\title{
Physician and Patient Perspective to Weight Gain in Pregnancy
}

\section{Tate $\mathrm{L}^{* 1}$ and Greene $\mathrm{R}^{2}$}

${ }^{1}$ University College Cork, Ireland

${ }^{2}$ Director of the National Perinatal Perinatal Epidemiology Centre and Professor of Clinical Obstetrics, University College Cork, Ireland

*Corresponding author: Tate L, University College Cork, Ireland, Tel: 00353872608832, E-mail: Lornapt1@ gmail.com

Citation: Tate L, Greene R (2017) Physician and Patient Perspective to Weight Gain in Pregnancy. J Obes Overweig 3(2): 203. doi: 10.15744/2455-7633.3.203

Received Date: September 20, 2017 Accepted Date: November 28, 2017 Published Date: November 30, 2017

\begin{abstract}
Background: Gaining weight outside of the Institute of Medicine guidelines puts a woman and her foetus at an increased risk. Limited or incorrect information is being provided to women during antenatal care. Research shows that physicians do not perceive this as an important issue.
\end{abstract}

Aims: We aimed to assess both physician and patient perspective to weight gain in pregnancy as well as assessing physicians current practice to weight management.

Methods: Quantitative cross-sectional study. Data collected by paper and online questionnaires.

Results: $71 \%$ of women want to be given a target weight to gain during pregnancy; $87 \%$ want to be told if they are gaining an inappropriate amount of weight. $87.5 \%$ of physicians believe that weight management is important; $31.2 \%$ advise patients about weight gain.

Conclusion: Women want discussions about weight gain to be an integral part of their antenatal care. Physicians think weight management is an important part of antenatal care however they do not routinely discuss it with patients.

Keywords: Physician; Patient; Pregnancy; Weight gain

List of abbreviations: IOM: Institute of Medicine; BMI: Body Mass Index; CUMH: Cork University Maternity Hospital; NCHDs: Nonconsultant house doctors

\section{Introduction}

According to the Institute of Medicine (IOM) gestational weight gain (GWG) is a unique and complex biological phenomenon that supports the growth and development of the foetus [1]. The total weight gained during pregnancy is multifactorial including the weight of the foetus, an increase in breast size, the placenta and amniotic fluid, as well as physiological changes including an increase in blood and fluid volume. These are intrinsic factors however they can be influenced by extrinsic factors such as diet, exercise and stress. The IOM GWG recommendations provide a guidance for both physicians and patients as to what is the recommended weight to gain depending on prepregnancy Body Mass Index (BMI). These guidelines suggest that women who are underweight (BMI <18.5) should gain 28-40 pounds (12-18 kg), normal weight (BMI 18.5-24.9) should gain 25-35 pounds (11-16 $\mathrm{kg}$ ), overweight (BMI 25-29.9) should gain 15-25 pounds (7-11 kg), obese (BMI>30) should gain 11-20 pounds (4.5-9 kg) [1]. In line with these guidelines, physicians are able to advise women what is the appropriate weight to gain in pregnancy.

Research has shown that up to $40 \%$ of women with a normal pre-pregnancy weight and $60 \%$ of overweight/obese women are gaining weight outside the current IOM guidelines [2]. The consequences of gaining excess weight during pregnancy are detrimental to both mother and foetus with maternal complications including an increased risk for gestational diabetes [3], hypertension [4], pre-eclampsia [5], labour and delivery complications [1], and an increased risk of weight retention postpartum [5]. The foetus is also at an increased risk for neonatal mortality, macrosomia [6], neonatal hypoglycaemia [7], as well as an increased incidence of obesity [7].

Receiving GWG advice is currently not mandatory, with $24 \%$ [8] to $41.7 \%$ [9] of women receiving advice from their health care provider. Of the $24 \%$ who receive advice, only $31 \%$ of these were within the IOM guidelines [ 8 ], meaning $69 \%$ of women 
received GWG that was not in accordance with guidelines. Women feel like they are not receiving sufficient advice about weight management in pregnancy [9].

Physicians feel there are several barriers to providing women with adequate advice [10]. Physicians don't perceive GWG as a significant matter. Out of a list of eleven issues that occur during pregnancy, 'appropriate weight management' ranked between four and nine [11]. Health care providers don't believe that they can effectively counsel women about weight management as they think family and cultural influences play a bigger part in a women's weight during pregnancy [12]. Physicians believe women can be influenced to 'eat for two' and not exercise if this fits with their family traditions [11]. Other perceived barriers to effective counselling include weight being a sensitive subject [11]. Physicians don't want to label a woman as being verweight/obese or tell her 'she is fat' [11]. The weighing scales is a major barrier to effective counselling about GWG, some physicians now use their 'clinical judgement' when evaluating a woman's weight [13]. Physicians don't routinely monitor a woman's weight as it is not 'in fashion' or 'in vogue' [13]. By estimating or inaccurately evaluating a woman's weight she may be given an incorrect pre-pregnancy BMI leading to inappropriate GWG advice.

Obesity is one of the most rapidly evolving epidemics in Ireland with The World Health Organisation (WHO) predicting that Ireland will be one of the fattest nations in the European Union [14]. The WHO research suggests that up to 85\% of Irish women will be overweight by 2035 [14]. This means that the percent of women who are starting pregnancy already overweight will drastically increase [14]. From this data we can see that it is imperative that healthcare professionals ensure they utilise the duration of pregnancy to try help women control their weight gain and also to understand more about living a healthy lifestyle. This will benefit the woman, her foetus and also society as a whole. There is currently no literature assessing physician's current practice in Ireland regarding advice about GWG, or literature looking at women's perspective of the issue.

\section{Materials and Methods}

A cross-sectional study was conducted in Cork University Maternity Hospital. A paper based questionnaire was handed out to women who presented for their booking visit in CUMH between May and July 2016. One hundred women agreed to participate in the study. Consultants and NCHDs were provided with an online questionnaire via SurveyMonkey. Due to poor response a paper based questionnaire was provided. The response rate was improved for the paper based questionnaire due to the researcher being present and able to assist with any questions the participants may have.

The inclusion criteria for patients were as follows; women who were attending their first antenatal booking visit, the ability to read English proficiently to complete the questionnaire, and consent to participate in the study. Consultants and NCHDs who were working in the area of Obstetrics and Gynaecology in CUMH were invited to participate in the study.

The results of the study were analysed using IBM-SPSS version 22. The main method of analysing the data was by descriptive statistics. By assessing the frequencies, we looked at the main characteristics of the study from both the physician and patient questionnaires (e.g. patients age, weight, number of pregnancies, physicians job title). Chi square testing was used to look for statistical significance among several variables. Fisher exact tests were also used in the analysis of contingency tables when the sample size for the question was small.

The patient questionnaire was validated by conducting a pilot test prior to the main data collection. The main questions from the patient questionnaire were as follows; 'Would you like your doctor to give you a target for weight gain during pregnancy?', 'Would you like to be weighed frequently during pregnancy to monitor your weight?', 'Would you like to be told that you are gaining too much or too little weight during pregnancy?', 'Do you think information about weight management, diet and exercise should be given during your antenatal appointments?' (Appendix 1). The physician's questionnaire was also validated by conducting a pilot test. The physician's questionnaire mainly looked at physician's current understanding and practice regarding weight management in pregnancy (Appendix 2).

\section{Results}

\section{Current Knowledge}

Table 1 shows that $41 \%(n=41)$ of women reported knowing how much weight to gain during pregnancy with two thirds $(n=27)$ being correctly able to identify weight gain in accordance with the IOM guidelines. $45 \%$ ( $n=45)$ indicated they knew the complications of gaining too much weight during pregnancy, however the majority of women were only able to identify one complication. $77.8 \%(n=35)$ indicating gestational diabetes mellitus as a complication. Other less common complications listed included hypertension/pre-eclampsia $(n=15)$ and labour or delivery difficulty $(n=10)$.

Women who indicated they knew how much weight to gain were also more likely to indicate knowing the complications associated with gaining too much weight $(\mathrm{p}<.023) .58 .5 \%(\mathrm{n}=24)$ of women who knew how much weight to gain also selected that they knew the complications of gaining too much weight.

Sixteen percent $(n=16)$ of the population currently believe that they are eating for two during pregnancy. Fifty percent of women who perceive their weight as obese believe they are eating for two, compared to only $9.4 \%$ of women who perceive their weight as normal. There is statistical significance between a woman's weight perception and if she believed she was eating for two ( $p<.031$ ). 


\begin{tabular}{|c|c|c|}
\hline \multicolumn{3}{|c|}{ Frequency results of patient questionnaire } \\
\hline & Number (n) & Percent $(\%)$ \\
\hline \multicolumn{3}{|l|}{ Weight perception } \\
\hline Underweight & 1 & 1 \\
\hline Normal weight & 64 & 64.6 \\
\hline Overweight & 30 & 30.4 \\
\hline Obese & 4 & 4 \\
\hline \multicolumn{3}{|c|}{ Weight gain knowledge } \\
\hline Yes & 41 & 41 \\
\hline No & 59 & 59 \\
\hline \multicolumn{3}{|l|}{ Target weight gain } \\
\hline Yes & 71 & 71 \\
\hline No & 29 & 29 \\
\hline \multicolumn{3}{|l|}{ Weighed frequently } \\
\hline Yes & 74 & 74.7 \\
\hline No & 25 & 25.3 \\
\hline \multicolumn{3}{|c|}{ Told if gaining too much/little } \\
\hline Yes & 87 & 87 \\
\hline No & 13 & 13 \\
\hline \multicolumn{3}{|c|}{ Knows complications } \\
\hline Yes & 45 & 45 \\
\hline No & 55 & 55 \\
\hline \multicolumn{3}{|c|}{ Discuss complications } \\
\hline Yes & 68 & 68.7 \\
\hline No & 31 & 31.3 \\
\hline \multicolumn{3}{|c|}{ Sensitive and should be avoided } \\
\hline Yes & 18 & 19.6 \\
\hline No & 78 & 80.4 \\
\hline \multicolumn{3}{|c|}{$\begin{array}{l}\text { Information provided about } \\
\text { weight, diet, exercise }\end{array}$} \\
\hline Yes & 91 & 91 \\
\hline No & 9 & 9 \\
\hline \multicolumn{3}{|c|}{ Added stress/anxiety } \\
\hline Yes & 33 & 33 \\
\hline No & 67 & 67 \\
\hline \multicolumn{3}{|l|}{ Eating for two } \\
\hline Yes & 16 & 16 \\
\hline No & 84 & 84 \\
\hline
\end{tabular}

\section{What women want from physicians}

Table 1 highlights that $71 \%(n=71)$ of women would like to be given a target weight gain during their pregnancy, $74.7 \%(n=74)$ of women would like to be weighed frequently throughout their pregnancy to monitor their weight. Eighty-seven percent $(\mathrm{n}=87)$ of women want to be told if they are gaining too much or too little weight during their pregnancy. We previously saw that only $45 \%(n=45)$ of women knew the complications associated with gaining too much weight, yet $68.7 \%(n=68)$ of women want these complications to be discussed at their antenatal appointments. Ninety-one percent $(n=91)$ of women want to receive more information about weight management, diet and exercise during pregnancy. Eighty point four percent ( $\mathrm{n}=78$ ) of women don't think discussing weight gain is sensitive and that it should be avoided. Sixty-seven percent $(n=67)$ of women are not of the opinion that discussing weight gain causes them any added stress or anxiety.

\section{Physician's results}

The physician demographics show that $43.8 \%(n=7)$ of respondents were Consultants and 56.3\% ( $n=9)$ were NCHDs. Table 2 shows how much weight physicians think women should gain during pregnancy depending on their pre-pregnancy BMI (normal 
weight, overweight, obese). When these answers were further analysed it showed that only $6.3 \%(n=1)$ of physicians were correctly able to identify how much weight a woman with a normal BMI should gain (25-35lbs), 12.5\% ( $\mathrm{n}=2$ ) of physicians correctly identified how much weight a woman who is overweight or obese should gain (15-25lbs and 10-20lbs respectively). Only one physician was able to correctly identify what weight a woman should gain in all three categories (normal weight, overweight and obese). No physician weighed their patients at every visit with $87.5 \%(n=14)$ indicating that they only weigh their patients at the booking visit. Furthermore $87.5 \%(n=14)$ of physicians believe that weight management is an important part of pregnancy but only $31.2 \%(n=5)$ will advise women on how much weight to gain. While $56.3 \%(n=9)$ will give advice about living a healthy lifestyle, diet and exercise.

\begin{tabular}{|c|c|c|}
\hline \multicolumn{3}{|c|}{ Frequency results of physician questionnaire } \\
\hline & Number (n) & Percent (\%) \\
\hline \multicolumn{3}{|l|}{ Job Title } \\
\hline Consultant & 7 & 43.8 \\
\hline NCHD & 9 & 56.3 \\
\hline \multicolumn{3}{|l|}{ Normal BMI } \\
\hline $10-20 \mathrm{lbs}$ & 7 & 43.8 \\
\hline $15-25 \mathrm{lbs}$ & 7 & 43.8 \\
\hline $25-35 \mathrm{lbs}$ & 1 & 6.3 \\
\hline $30-40 \mathrm{lbs}$ & 1 & 6.3 \\
\hline \multicolumn{3}{|l|}{ Overweight BMI } \\
\hline $10-20 \mathrm{lbs}$ & 13 & 81.3 \\
\hline $15-25 \mathrm{lbs}$ & 2 & 12.5 \\
\hline $20-30 \mathrm{lbs}$ & 0 & 0 \\
\hline $25-35 \mathrm{lbs}$ & 0 & 0 \\
\hline $30-40 \mathrm{lbs}$ & 1 & 6.3 \\
\hline \multicolumn{3}{|l|}{ Obese BMI } \\
\hline $5-15 \mathrm{lbs}$ & 13 & 81.3 \\
\hline $10-20 \mathrm{lbs}$ & 2 & 12.5 \\
\hline $15-25 \mathrm{lbs}$ & 0 & 0 \\
\hline $20-30 \mathrm{lbs}$ & 0 & 0 \\
\hline $25-35 \mathrm{lbs}$ & 1 & 6.3 \\
\hline \multicolumn{3}{|l|}{ When do you weigh patients } \\
\hline Booking & 14 & 87.5 \\
\hline Every visit & 0 & 0 \\
\hline Booking and $3^{\text {rd }}$ Trimester & 2 & 12.5 \\
\hline \multicolumn{3}{|l|}{ Advise weight gain } \\
\hline Yes & 5 & 31.2 \\
\hline No & 11 & 68.8 \\
\hline \multicolumn{3}{|l|}{ Sensitive Issue } \\
\hline Yes & 11 & 68.8 \\
\hline No & 5 & 31.3 \\
\hline \multicolumn{3}{|l|}{$\begin{array}{l}\text { Is weight management } \\
\text { important }\end{array}$} \\
\hline Yes & 14 & 87.5 \\
\hline No & 2 & 12.5 \\
\hline \multicolumn{3}{|l|}{ Advice about diet/exercise } \\
\hline Yes & 9 & 56.3 \\
\hline No & 7 & 43.8 \\
\hline \multicolumn{3}{|l|}{ More resources } \\
\hline Yes & 14 & 87.5 \\
\hline No & 2 & 12.5 \\
\hline
\end{tabular}

Table 2: Physician frequencies 
Physicians provided written reasons as to why they do give patients a target weight gain during pregnancy. These included:

- Physicians not knowing the evidence behind it

- Not wanting to upset the patients

- Low priority/do not think it is relevant/not my business

- Patients will generally ignore advice, especially if overweight

- Not enough time

The reasons why physicians think discussing weight is a sensitive issue include:

- Upsets the patients

- Patients don't like to hear they are overweight

- Women become defensive when discussing weight

- The women take it very personally

- Stigma around 'fat-shaming' a woman

$62.5 \%$ of physicians are not using any source for advice about weight gain in pregnancy. Of those who do use a source, there is an abundance of different sources used, including:

- Institute of Medicine guidelines (1)

- $\operatorname{RCOG}(1)$

- NICE guidelines (1)

- Textbooks (1)

$87.5 \%$ of physicians feel they would benefit from additional resources to help them advise women about how much weight to gain during pregnancy. A higher percentage of NCHDs (100\%) want more resources when compared to consultants (71.4\%).

\section{Discussion}

Weight gain during pregnancy is very important to ensure good health for both the mother and her foetus, however gaining too much has a myriad of consequences for both. From this research we see that women want weight gain to be an integral part of their antenatal care. It is imperative that we as healthcare professionals ensure that we provide women with adequate information to have a healthy and happy pregnancy.

In this study we aimed to look at what women currently know about weight gain in pregnancy and we also tried to understand what more they would like to know. In relation to a woman's current understanding of weight gain in pregnancy we found out that the majority of women do not know how much weight to gain in pregnancy. Over half of women who were on their second pregnancy or more indicated that they didn't know how much weight to gain. This may imply that they were not provided with information about weight gain in their previous pregnancies. Furthermore, those who indicated they knew how much to gain, were not always correct with an overwhelming 1/3rd of women being wrong. Again, this is concerning as women may be receiving inaccurate information from physicians.

We looked at women's understanding of the complications of gaining too much weight in pregnancy. The minority of women knew the complications associated with excess GWG and even a significant proportion of those who did, were only able to identify gestational diabetes or hypertension. Only a small proportion of women specifically identified that gaining too much weight may affect the foetus in some way and no woman identified that gaining too much weight can have serious long term effects on her health. This emphasizes that there is a flaw in the information that we provide women during pregnancy. Over $68 \%$ of women want these complications to be discussed with them, yet for some reason physicians fail to do so, be it they don't perceive it as important or needed by the patient. Whilst a lot of the literature looks at the sensitivity of talking about weight, not much looks at the importance of discussing the complications that can occur when a woman gains too much. There is a gap in the current literature regarding this information and future research could look into the reasons behind this.

One previous study reported that only $51 \%$ of women want to receive more information about weight gain in pregnancy [10] however from our results we see that $91 \%$ of women want to receive this advice. With such a high percentage of women wanting information it would be absurd not to provide it. We are doing these women a disservice by ignoring their enthusiasm and desire to learn and manage their weight. Old literature suggests that physicians aren't comfortable discussing weight gain as it is a sensitive issue, our results show $78 \%$ of women don't think weight gain is a sensitive issue that should be avoided. This data suggests that there has been a change in women's views of weight gain over time and that possibly women are becoming more conscious of weight management. As healthcare professionals we should be seizing every opportunity to aid women with weight management. Women want more personalised weight advice [10] rather than generic pamphlets or hand-outs. We saw that an overwhelming majority of women want to be given a specific target weight gain during pregnancy. When a woman is provided with accurate advice she is much more likely to gain within the recommended limits. One study documented that $85 \%$ of women who were given advice about weight gain, gained within the IOM guidelines [9]. With results like that, it is hard to believe that more physicians aren't providing women with adequate advice.

Women who start pregnancy as overweight or obese are more likely to gain weight outside of the guidelines for their BMI. There was an association between weight perception and 'eating for two' with women who perceive themselves as overweight or obese 
being more likely to believe they are eating for two. This can be detrimental to a woman's health as she will significantly increase her calorie intake leading to excessive weight gain. Years of research suggests that a woman only needs to increase her calorie intake by approximately 300 calories which is the equivalent of a slice of bread and a tablespoon of peanut butter. These needs to be highlighted to women as the majority are unaware of this.

Women who perceive themselves to be overweight or obese are more likely to think they are eating for two compared to women who perceive themselves as normal weight. With up to $60 \%$ of overweight/obese women gaining outside the recommended guidelines it is imperative that these women receive advice tailored to them. Physicians have said that overweight/obese women are more likely to become defensive and not want to hear about weight gain but these are the women who need to hear about it the most. They are at an increased risk of developing severe complications due to the excess weight gain. As healthcare professionals we must not ignore the opportune time that pregnancy offers to help a woman gain control over her weight. 100\% of obese women and $70 \%$ of overweight women want to be weighed frequently. This highlights that physicians shouldn't think these women are more sensitive to the issue or that they will be more offended than normal weight women.

The literature indicated that physicians don't perceive weight gain and weight management as an important topic [11]. From our data we see that this is not the case with the physicians we surveyed as the majority believe that weight management is an important issue. Despite thinking it is an important issue we do see that physicians agree with the literature, in saying that they don't think they can effectively counsel women about weight gain [12]. We saw this when the physicians mentioned that 'patient's will ignore advice generally'. Even though the majority think that weight management is important, only the minority will actually advise women on how much weight to gain during pregnancy.

There are many barriers to effective counselling of women about weight gain during pregnancy. Over $68 \%$ of physicians think that it is a sensitive issue compared to only $18 \%$ of women. This highlights that there is a discrepancy between what physicians and patients perceive regarding weight gain during pregnancy. The physicians are fearful of upsetting the patient, especially those who are overweight or obese at the start of pregnancy, they believe that women can become quite defensive when the topic of weight gain is brought up in the consultation and finally physicians are afraid of the stigma associated with 'fat-shaming' a woman based on her weight. It is unfortunate that women are being denied valuable information about weight gain because of physician's fear of upsetting women. As we saw, $71 \%$ of women want a target weight to gain during pregnancy and a further $91 \%$ of women want advice about weight management including diet and exercise. It must be highlighted to physicians the discrepancy between what women want and what physicians think they want.

\section{Conclusion}

We found incongruence between physician and patient perception of weight gain in pregnancy. Most women want to be given a target weight to gain during pregnancy however most physicians don't tell patients how much weight to gain. The results of this study highlight that there is an urgent need to reform the way weight is managed during pregnancy. Physicians need to start discussing weight with patients more frequently and ensure that it is a significant part of their antenatal care especially women who are already overweight or obese before starting pregnancy. More resources needed to be provided to physicians so that they are able to provide patients with accurate advice about weight gain and to ensure that the weight is in accordance with the IOM guidelines. Further research will be required to assess the progress made regarding weight management during pregnancy.

\section{References}

1. Moore Simas TA, Waring ME, Sullivan GMT, Liao X, Rosal MC, et al. (2013) Institute of Medicine 2009 Gestational Weight Gain Guideline Knowledge: Survey of Obstetrics/Gynecology and Family Medicine Residents of the United States. Birth 40: 237-46.

2. Chu SY, Callaghan WM, Bish CL, D’Angelo D (2009) Gestational weight gain by body mass index among US women delivering live births, 2004-2005: fueling future obesity. Am J Obs Gynecol 200: 271.e1-7.

3. Dabelea D, Snell-Bergeon JK, Hartsfield CL, Bischoff KJ, Hamman RF, et al. (2005) Increasing prevalence of gestational diabetes mellitus (GDM) over time and by birth cohort: Kaiser Permanente of Colorado GDM Screening Program. Diabetes care 28: 579-84.

4. Gaillard R, Bakker R, Willemsen SP, Hofman A, Steegers EA, et al. (2011) Blood pressure tracking during pregnancy and the risk of gestational hypertensive disorders: The Generation R Study. Eur Heart J 32: 3088-97.

5. Nohr EA, Vaeth M, Baker JL, Sorensen T, Olsen J, et al. (2008) Combined associations of prepregnancy body mass index and gestational weight gain with the outcome of pregnancy. Am J Clini Nutri 87: 1750-9.

6. Chen A, Feresu SA, Fernandez C, Rogan WJ (2009) Maternal obesity and the risk of infant death in the United States. Epidemiology 20: 74-81.

7. Viswanathan M, Siega-Riz AM, Moos MK, Deierlein A, Mumford S, et al. (2008) Outcomes of maternal weight gain. Evid Rep Technol Assess (Full Rep): 1-223.

8. Arinze NV, Karp SM, Gesell SB (2016) Evaluating Provider Advice and Women's Beliefs on Total Weight Gain During Pregnancy. J Immigr Minor Health 18: $282-6$.

9. McDonald SD, Pullenayegum E, Taylor VH, Lutsiv O, Bracken K, et al. (2011) Despite 2009 guidelines few women report being counseled correctly about weight gain during pregnancy. Am J Obstet Gynecol 205: 333.e1-6.

10. Brown A, Avery A (2012) Healthy weight management during pregnancy: what advice and information is being provided. J Hum Nutr Diet 25: $378-87$.

11. Chang T, Llanes M, Gold KJ, Fetters MD (2013) Perspectives about and approaches to weight gain in pregnancy: a qualitative study of physicians and nurse midwives. BMC Pregnancy Childbirth 13: 47

12. Stotland NE, Gilbert P, Bogetz A, Harper CC, Abrams B, et al. (2010) Preventing excessive weight gain in pregnancy: how do prenatal care providers approach counseling? J Womens Health 19: 807-14. 
13. Knight-Agarwal CR, Kaur M, Williams LT, Davey R, Davis D (2014) The views and attitudes of health professionals providing antenatal care to women with a high BMI: a qualitative research study. Women Birth 27: 138-44.

14. Dohrman KR, Lederman SA (1986) Weight Gain in Pregnancy Dohrmann. J Obst Gynecol Neonatal Nursing 15: 446-53.

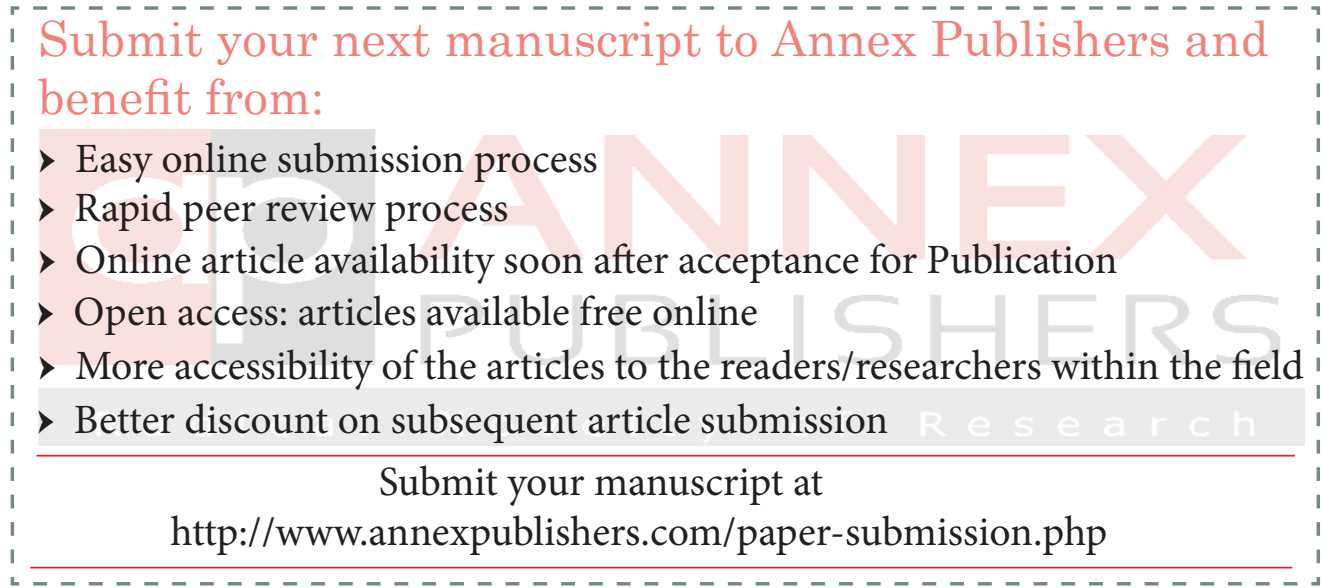

